\title{
The rational points close to a curve II
}

\author{
by
}

\author{
M. N. HuXley (Cardiff)
}

1. Introduction. Halberstam and Roth's work on gaps between $k$-free numbers [2] rests on studying the pairs of integers $m, q$ with

$$
N<m^{k} q \leq N+H
$$

(and $m$ prime; but the primality is hardly used), for given $N$ and $H$, with $m, q$ bounded below by powers of $N$. A geometrical interpretation of (1.1) is that $(m, q)$ is an integer point close to the curve $y=N / x^{k}$. The general problem of bounding the number of integer points close to a curve $y=f(x)$ was discussed in (for example) Huxley [3], Filaseta and Trifonov [1], and Huxley and Sargos [6]. The key idea is to write down an integer determinant corresponding to $r$ solutions of (1.1), which is small by the approximation property. The determinant is zero on major arcs, regions where $f(x)$ is approximated by a polynomial of small degree with rational coefficients. Rational points with a common denominator were studied in [4], using the duality of points and lines in the projective plane.

The inequality

$$
0<m^{k} q-n^{k} r \leq H
$$

is related to the distribution of gaps between $k$-free numbers studied in [5]. The rational point $(m / n, r / q)$ is close to the curve $y=x^{1 / k}$. Having four variables should make matters easier, but the determinants have large order. A symmetry-breaking variation is to ask for points $(m, r / q)$ close to a curve $y=f(x)$, with the parameter $n$ absorbed into the function $f(x)$.

THEOREM 1. Let $F(x)$ be a real function three times continuously differentiable on an interval $I \subset[1 / 2,2]$, with

$$
\left|F^{(i)}(x)\right| \leq C^{i+1} \lambda
$$

for $i=1,2,3$,

2000 Mathematics Subject Classification: 11J25, 11P21, 11K38. 


$$
\left|F^{(i)}(x)\right| \geq \lambda / C^{i+1}
$$

for $i=1,2$, and

$$
\left|3 F^{\prime \prime}(x)^{2}-2 F^{\prime}(x) F^{(3)}(x)\right| \geq \lambda^{2} / C^{6} .
$$

Let $M$ and $Q$ be large positive integers. Given $\delta$ with $0 \leq \delta<1 / 2$, let $S$ be the set of rational points of the form $(m / n, r / q)$ with $m, n, r$ and $q$ integers with $(m, n)=1,(r, q)=1,1 \leq m \leq M, 1 \leq n \leq M, m / n$ in $I, 1 \leq q \leq Q$, and

$$
\left|F\left(\frac{m}{n}\right)-\frac{r}{q}\right| \leq \frac{\delta}{Q^{2}} \leq \delta \lambda .
$$

Then $R$, the size of $S$, satisfies

$$
R=O\left(\left(C^{6} \delta^{1 / 4} M^{2}+C^{2}\left(\lambda M^{2} Q^{2}\left(\delta M^{2}+C^{2}\right)\right)^{1 / 3}\right)\left(C \lambda M Q^{2}\right)^{\varepsilon}\right)
$$

for any $\varepsilon>0$. The implied constant depends on $\varepsilon$, but not on $C, \delta, \lambda, M$ or $Q$.

THEOREM 2. Let $f(x)$ be a real function three times continuously differentiable on an interval $I$ of length $M$ with integer endpoints. Suppose that on I we have

$$
\left|f^{(i)}(x)\right| \leq C^{i+1} \lambda / M^{i}
$$

for $i=1,2,3$,

$$
\left|f^{(i)}(x)\right| \geq \lambda /\left(C^{i+1} M^{i}\right)
$$

for $i=1,2$, and

$$
\left|3 f^{\prime \prime}(x)^{2}-2 f^{\prime}(x) f^{(3)}(x)\right| \geq \lambda^{2} /\left(C^{6} M^{4}\right) .
$$

Given $\delta$ with $0 \leq \delta<1 / 2$, let $S$ be the set of rational points of the form $(m, r / q)$ with $m$ an integer in $I, r$ and $q$ integers with $(r, q)=1,1 \leq q \leq Q$, and

$$
\left|f(m)-\frac{r}{q}\right| \leq \frac{\delta}{Q^{2}} \leq \delta \lambda
$$

Then $R$, the size of $S$, satisfies

$$
R=O\left(\left(C^{6} \delta^{1 / 4} M+C^{2}\left(\lambda M Q^{2}(\delta M+C)\right)^{1 / 3}\right)\left(C \lambda M Q^{2}\right)^{\varepsilon}\right)
$$

for any $\varepsilon>0$. The implied constant depends on $\varepsilon$, but not on $C, \delta, \lambda, M$ or $Q$.

The expected number of rational points is $O\left(\delta M^{2}\right)$ in Theorem 1, $O(\delta M)$ in Theorem 2. Lemmas 1.1 and 1.2 below give a very simple argument which gets the expected upper bounds $O\left(\delta M^{2}\right)$ and $O(\delta M)$ for a smaller range of $Q$ under a weaker hypothesis on the derivatives of the function. Our theorems extend the range for $Q$, but they give weaker upper bounds, and they require stronger conditions. For the theorems we consider major and minor arcs. 
The major arcs correspond to linear fractional approximations to $F(x)$. The condition (1.6) becomes stronger as we increase $Q$, corresponding to higher terms in the Taylor expansion of $F(x)$. To extend our results to larger $Q$ we need Padé approximants of higher degree. However Lemma 3.5 depends on the Padé approximant having only one pole. The equation (1.2) appears in Swinnerton-Dyer's method for integer points close to curves ([7], (16), [3], (5.8)]) with $Q$ near $M^{2}$ in size, corresponding to derivatives of the fifth order or more.

Using Theorem 1 we can establish the asymptotic formula of [5],

$$
\sum_{i}\left(s_{i+1}-s_{i}\right)^{\gamma} \simeq \beta(\gamma) N,
$$

where the sum is over pairs of consecutive square-free numbers $s_{i}, s_{i+1}$ with $s_{i+1} \leq N$, in a longer range $\gamma<59 / 16=3.6875$; the range in [5] was $\gamma<11 / 3$.

We now give the easy bounds that correspond to Theorems 1 and 2 .

Lemma 1.1 (small Q). Suppose that

$$
C^{2} \lambda Q^{2} \geq 1
$$

and that (1.3) and (1.4) hold for $i=1$. Then the number $R$ of points, defined as in Theorem 1, satisfies

$$
R \leq 8 C^{4} \delta M^{2}+4 C^{2} \lambda Q^{2} .
$$

Proof. The rationals $r / q$ lie in an interval of length at most

$$
2 \delta / Q^{2}+2 \max \left|F^{\prime}(x)\right| \leq 2 C^{2} \lambda+2 \delta / Q^{2} \leq 3 C^{2} \lambda,
$$

so the number of possible rational numbers $r / q$ is

$$
\leq 1+3 C^{2} \lambda Q^{2} \leq 4 C^{2} \lambda Q^{2} .
$$

If the same rational $r / q$ occurs for $k$ consecutive fractions of the Farey sequence $\mathcal{F}(M)$, then

$$
\frac{2 \delta}{Q^{2}} \geq \frac{k-1}{M^{2}} \min \left|F^{\prime}(x)\right| \geq \frac{(k-1) \lambda}{C^{2} M^{2}},
$$

so that

$$
k \leq \frac{2 C^{2} \delta M^{2}}{\lambda Q^{2}}+1
$$

We multiply (1.13) by (1.14) to obtain the result of Lemma 1.1.

Lemma 1.2 (small Q). Suppose that

$$
C^{2} \lambda Q^{2} \geq 1
$$


and that (1.8) and (1.9) hold for $i=1$. Then the number $R$ of points, defined as in Theorem 2, satisfies

$$
R \leq 6 C^{4} \delta M+3 C^{2} \lambda Q^{2} .
$$

Proof. As Lemma 1.1.

We give the proof of Theorem 1 in full. The proof of Theorem 2 is analogous, and simpler in one or two places.

2. Major and minor arcs. We use the cross-ratio

$$
\left(x_{1}, x_{2} ; x_{3}, x_{4}\right)=\frac{x_{1}-x_{3}}{x_{3}-x_{2}} \cdot \frac{x_{4}-x_{2}}{x_{1}-x_{4}} .
$$

LEMMA 2.1 (cross-ratio invariance). Suppose that $F(x)$ is a real function three times continuously differentiable on an interval $I$, on which, for some positive constants $C$ and $\lambda$,

$$
\left|F^{(i)}(x)\right| \leq C^{i+1} \lambda
$$

for $i=1,2,3$, and

$$
\left|F^{\prime}(x)\right| \geq \lambda / C^{2}
$$

Let $x_{1}, \ldots, x_{4}$ be distinct points in $I$ with $\left|x_{i}-x_{j}\right| \leq K$, where

$$
K \leq 1 /\left(4 C^{5}\right) .
$$

Let $y_{i}=F\left(x_{i}\right)$. Then

$$
\frac{1}{1+E} \leq \frac{\left(y_{1}, y_{2} ; y_{3}, y_{4}\right)}{\left(x_{1}, x_{2} ; x_{3}, x_{4}\right)} \leq 1+E,
$$

where

$$
E=128 C^{10} K^{2} \text {. }
$$

Proof. The statement of the lemma is invariant under translation, so we may take $x_{1}=0$. Let $\alpha=F^{\prime}(0), \beta=\frac{1}{2} F^{\prime \prime}(0)$. By Taylor's theorem

$$
F^{\prime}(x)-\alpha-2 \beta x=\frac{1}{2} x^{2} F^{(3)}(\xi)
$$

for some $\xi$ between 0 and $x$. Hence for $|x| \leq K$,

$$
\left|F^{\prime}(x)-\alpha-2 \beta x\right| \leq \frac{1}{2} C^{4} \lambda K^{2} .
$$

Let

$$
\theta_{i j}=\frac{y_{j}-\alpha x_{j}-\beta x_{j}^{2}-\left(y_{i}-\alpha x_{i}-\beta x_{i}^{2}\right)}{x_{j}-x_{i}} .
$$

By Cauchy's mean value theorem

$$
\theta_{i j}=F^{\prime}(\xi)-\alpha-2 \beta \xi
$$


for some $\xi$ between $x_{i}$ and $x_{j}$. By (2.3)

$$
\frac{y_{j}-y_{i}}{x_{j}-x_{i}}=\alpha+\beta\left(x_{i}+x_{j}\right)+\theta_{i j}
$$

with

$$
\left|\theta_{i j}\right| \leq \frac{1}{2} C^{4} \lambda K^{2}
$$

Also by (2.3), for $|x| \leq K$,

$$
|\alpha+2 \beta x| \leq C^{2} \lambda+\frac{1}{2} C^{4} \lambda K^{2} \leq 2 C^{2} \lambda .
$$

We can now compute

$$
\begin{aligned}
\mid \frac{y_{1}-y_{3}}{x_{1}-x_{3}} \cdot \frac{y_{4}-y_{2}}{x_{4}-x_{2}}- & \alpha^{2}-\alpha \beta\left(x_{1}+x_{2}+x_{3}+x_{4}\right) \mid \\
= & \mid \beta^{2}\left(x_{1}+x_{3}\right)\left(x_{2}+x_{4}\right)+\theta_{24}\left(\alpha+\beta\left(x_{1}+x_{3}\right)\right) \\
& +\theta_{13}\left(\alpha+\beta\left(x_{2}+x_{4}\right)\right)+\theta_{13} \theta_{24} \mid \\
\leq & \left(C^{3} \lambda K\right)^{2}+2 \cdot \frac{1}{2} C^{4} \lambda K^{2} \cdot 2 C^{2} \lambda+\left(\frac{1}{2} C^{4} \lambda \kappa^{2}\right)^{2} \\
\leq & 3 C^{6} \lambda^{2} K^{2}+\frac{1}{4} C^{8} \lambda^{2} K^{4} \leq 4 C^{6} \lambda^{2} K^{2},
\end{aligned}
$$

where we have used (2.1).

We also have

$$
\left|\alpha^{2}+\alpha \beta\left(x_{1}+x_{2}+x_{3}+x_{4}\right)\right| \geq|\alpha|^{2}\left(1-2 C^{5} K\right) \geq \frac{1}{2}|\alpha|^{2} \geq \lambda^{2} /\left(2 C^{4}\right),
$$

so

and similarly

$$
\frac{y_{1}-y_{3}}{x_{1}-x_{3}} \cdot \frac{y_{4}-y_{2}}{x_{4}-x_{2}}=\left(\alpha^{2}+\alpha \beta\left(x_{1}+x_{2}+x_{3}+x_{4}\right)\right) \kappa,
$$

$$
\frac{y_{3}-y_{2}}{x_{3}-x_{2}} \cdot \frac{y_{1}-y_{4}}{x_{1}-x_{4}}=\left(\alpha^{2}+\alpha \beta\left(x_{1}+x_{2}+x_{3}+x_{4}\right)\right) \mu,
$$

with

$$
|\kappa-1|,|\mu-1| \leq \frac{2 C^{4}}{\lambda^{2}} \cdot 4 C^{6} \lambda^{2} K^{2} \leq 8 C^{10} K^{2} \leq \frac{1}{2},
$$

by (2.1) again. Since

$$
\left(\frac{1+t}{1-t}\right)^{2}=1+\frac{4 t}{(1-t)^{2}} \leq 1+16 t
$$

for $0 \leq t \leq 1 / 2$, we have

$$
\frac{\kappa}{\mu}, \frac{\mu}{\kappa} \leq 1+128 C^{10} K^{2}
$$

which proves (2.2).

Lemma 2.2 (major/minor dichotomy). Suppose that

$$
\delta M^{2} \leq C^{2} \lambda Q^{2},
$$


and the conditions of Lemma 2.1 hold. Let $\left(x_{i}, r_{i} / q_{i}\right)$ be distinct points of $S$, with $x_{i}=m_{1} / n_{i}$. Suppose that

$$
K \leq 1 /\left(160 C^{7} \lambda M^{2} Q^{2}\right)^{1 / 3} .
$$

Then either

$$
\left(\frac{r_{1}}{q_{1}}, \frac{r_{2}}{q_{2}} ; \frac{r_{3}}{q_{3}}, \frac{r_{4}}{q_{4}}\right)=\left(x_{1}, x_{2} ; x_{3}, x_{4}\right)
$$

or

$$
\min _{i \neq j}\left|x_{j}-x_{i}\right| \leq 200 \delta C^{6} K^{4} \lambda M^{4} Q^{2} .
$$

Proof. We have

$$
\left|m_{j} n_{i}-m_{i} n_{j}\right| \leq K M^{2} .
$$

The cross-ratio $G=\left(x_{1}, x_{2} ; x_{3}, x_{4}\right)$ is a rational number with numerator and denominator numerically at most $K^{2} M^{4}$. Similarly

$$
\left|\frac{r_{j}}{q_{j}}-\frac{r_{i}}{q_{i}}\right| \leq C^{2} \lambda K+\frac{2 \delta}{Q^{2}} \leq 2 C^{2} \lambda K,
$$

since (2.4) gives

$$
K \geq 1 / M^{2} \geq \delta /\left(C^{2} \lambda Q^{2}\right) .
$$

The cross-ratio $H=\left(r_{1} / q_{1}, r_{2} / q_{2} ; r_{3} / q_{3}, r_{4} / q_{4}\right)$ is a rational number whose numerator and denominator are numerically at most $4 C^{4} \lambda^{2} K^{2} Q^{4}$. If $G \neq H$, then

$$
\left|\frac{G}{H}-1\right| \geq \frac{1}{4 C^{4} \lambda^{2} K^{4} M^{4} Q^{4}} .
$$

More accurately, we have

$$
\frac{r_{j}}{q_{j}}-\frac{r_{i}}{q_{i}}=y_{j}-y_{i}+\eta_{i j} \frac{\delta}{Q^{2}}
$$

with $\left|\eta_{i j}\right| \leq 2$. Since

$$
\left|\frac{y_{j}-y_{i}}{x_{j}-x_{i}}\right|=\left|F^{\prime}(\xi)\right| \geq \frac{\lambda}{C^{2}}
$$

for some $\xi$, we have

$$
\frac{r_{j}}{q_{j}}-\frac{r_{i}}{q_{i}}=\left(y_{j}-y_{i}\right)\left(1+\frac{\theta_{i j} \delta C^{2}}{\lambda Q^{2}\left|x_{j}-x_{i}\right|}\right)
$$

with $\left|\theta_{i j}\right| \leq 2$. By (2.5),

$$
128 C^{10} K^{2} \leq 1 /\left(100 C^{4} \lambda^{2} K^{4} M^{4} Q^{4}\right) .
$$

If (2.7) is false, then

$$
\frac{2 \delta C^{2}}{\lambda Q^{2}\left|x_{j}-x_{i}\right|} \leq \frac{1}{100 C^{4} K^{4} M^{4} Q^{4}} .
$$


Since

$$
\frac{(1+t)^{3}}{(1-t)^{2}} \leq 1+25 t
$$

for $0 \leq t \leq 1 / 2$, (2.8) cannot hold, and we deduce $G=H$, which is (2.6).

We define a major arc $J$ to be a subinterval of $I$ such that there are at least four points of $S$ with $m / n$ in $J$, and all points of $S$ with $m / n$ in $J$ have $r / q=G(m / n)$, for some linear fractional function

$$
G(x)=\frac{\alpha x+\beta}{\gamma x+\theta} .
$$

Subintervals of $I$ which are not major arcs are called minor arcs.

LEMma 2.3 (nested intervals). Let $J$ be a subinterval of $I$ of length $K$. Suppose that the conditions of Lemma 2.2 hold. Then either $J$ is a major arc, or the points of $S$ in $J$ lie in at most three subintervals of lengths at most $E K^{4}$, where

$$
E=800 \delta C^{6} \lambda M^{4} Q^{2} .
$$

Pr o of. The negation of the condition (2.7) of Lemma 2.2 can be written as

$$
\left|x_{j}-x_{i}\right| \geq K_{1} / 4=E K^{4} / 4 \text {. }
$$

If we cannot choose three points in $J$ such that (2.9) holds for each pair, then the points of $S$ in $J$ lie in at most two subintervals of length $K_{1} / 2$, and the lemma holds. Suppose that we can choose three points $P_{1}, P_{2}, P_{3}$ of $S$ in $J$ so that (2.9) holds for each pair. If there is another point $P_{4}$ of $S$ in $J$, then by Lemma 2.2, either $P_{4}$ lies on the linear fractional curve through $P_{1}$, $P_{2}, P_{3}$, or for some $i=1,2$, or 3 ,

$$
\left|x_{4}-x_{i}\right|<K_{1} / 4
$$

Hence the points of $S$ in $J$ either lie on the linear fractional curve through $P_{1}$, $P_{2}, P_{3}$, or they have $x$ in one of three intervals of length $K_{1} / 2$ with centres $x_{1}$, $x_{2}$, or $x_{3}$. Suppose that both possibilities occur: $P_{4}$ is on the linear fractional curve through $P_{1}, P_{2}, P_{3}$, but $P_{5}$ is not. Then $x_{5}$ is close to $x_{1}, x_{2}$, or $x_{3}$; suppose that $x_{5}$ is close to $x_{1}$, so that $\left|x_{5}-x_{1}\right|<K_{1} / 4$. Since $P_{5}$ is not on the linear fractional curve through $P_{2}, P_{3}, P_{4}$, we have (2.10) with $i=2,3$, or 5 , so $\left|x_{4}-x_{i}\right|<K_{1} / 2$ for $i=1,2$, or 3 . Hence if $J$ is not a major arc, then all points of $S$ in $J$ lie in one of three intervals of length $K_{1}=E K^{4}$, centres $x_{1}, x_{2}$, or $x_{3}$, which proves the lemma.

Lemma 2.4 (local structure of $S$ ). Let $J$ be a subinterval of I of length $K$. Suppose that the conditions of Lemma 2.2 hold, and

$$
K \leq K_{0}=\left(\frac{\min \left(80 \delta M^{2}, C\right)}{12800 \delta C^{7} \lambda M^{4} Q^{2}}\right)^{1 / 3} .
$$


Let

$$
L=3\left(\frac{\log 4 M}{\log 4}\right)^{\log 3 / \log 4} .
$$

Then the points of $S$ in $J$ lie in at most $L$ subintervals of $J$. Each subinterval either contains one point only of $S$, or it is a major arc.

Pr o of. Since $K \leq K_{0}$, in Lemma 2.3 we have by (2.11)

$$
K_{1} / K=E K^{3} \leq 800 \delta C^{6} \lambda M^{4} Q^{2} K_{0}^{3} \leq 1 / 16 .
$$

We can now iterate Lemma 2.3: in each of the three subintervals of length $K_{1}$, either all the points of $S$ lie on a linear fractional curve, or they lie within three subintervals of length $K_{2}=E K_{1}^{4}$, and so on. At the $r$ th step

$$
K_{r}=E K_{r-1}^{4}=E^{1+4+\ldots+4^{r-1}} K^{4^{r}}=\frac{\left(E K^{3}\right)^{4^{r} / 3}}{E^{1 / 3}} .
$$

We continue until $K_{r}<1 / M^{2}$. If $E<1 /\left(16 M^{2}\right)$, this occurs for $r=1$. If $E \geq 1 /\left(16 M^{2}\right)$, then by (2.12) we must have $K_{r}<1 / M^{2}$ for

$$
4^{r}>\frac{\log M}{\log 4}+1 \text {. }
$$

We take

$$
r=\left[\frac{1}{\log 4} \log \left(\frac{\log 4 M}{\log 4}\right)+1\right] .
$$

The number of subintervals is at most $3^{r} \leq L$. Since an interval of length $K_{r}<1 / M^{2}$ contains at most one point of $S$, we have the lemma.

3. Determinants related to Padé approximants. First we need some mean value results involving determinants. Symbols such as $C$ and $\delta$ do not necessarily carry the same meaning as in Theorem 1. I would like to thank my colleagues V. I. Bourenkov and A. M. Cohen for formulating Lemma 3.1, and for other valuable suggestions. Suppose that $a_{1}, \ldots, a_{r}$ are distinct real numbers in ascending order. Let $V\left(a_{1}, \ldots, a_{r}\right)$ denote the Vandermonde determinant

$$
V\left(a_{1}, \ldots, a_{r}\right)=\left|\begin{array}{ccccc}
a_{1}^{r-1} & a_{1}^{r-2} & \ldots & a_{1} & 1 \\
\ldots \ldots & \ldots & \ldots & \ldots & \ldots \\
a_{r}^{r-1} & a_{r}^{r-2} & \ldots & a_{r} & 1
\end{array}\right| .
$$

Lemma 3.1 (determinant mean value theorem). Let $E\left(a_{1}, \ldots, a_{r}\right)$ and $E^{\prime}\left(b_{1}, \ldots, b_{r-1}\right)$ denote the determinants of function values 


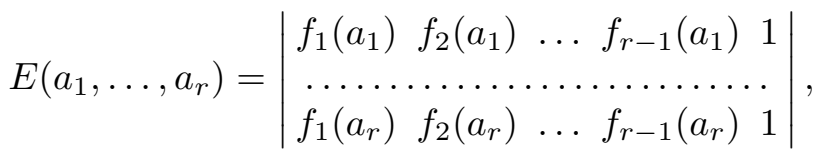

$$
\begin{aligned}
& E^{\prime}\left(b_{1}, \ldots, b_{r-1}\right)=\left|\begin{array}{cccc}
f_{1}^{\prime}\left(b_{1}\right) & f_{2}^{\prime}\left(b_{1}\right) & \ldots & f_{r-1}^{\prime}\left(b_{1}\right) \\
\ldots \ldots \ldots \ldots \ldots \ldots \ldots \ldots & \ldots \ldots \ldots \ldots \\
f_{1}^{\prime}\left(b_{r-1}\right) & f_{2}^{\prime}\left(b_{r-1}\right) & \ldots & f_{r-1}^{\prime}\left(b_{r-1}\right)
\end{array}\right|,
\end{aligned}
$$

where $f_{i}(x)$ are continuously differentiable functions. Then

$$
\frac{E\left(a_{1}, \ldots, a_{r}\right)}{V\left(a_{1}, \ldots, a_{r}\right)}=\frac{1}{(r-1) !} \cdot \frac{E^{\prime}\left(\alpha_{1}, \ldots, \alpha_{r-1}\right)}{V\left(\alpha_{1}, \ldots, \alpha_{r-1}\right)}
$$

for some $\alpha_{1}, \ldots, \alpha_{r-1}$ with

$$
a_{1}<\alpha_{1}<\alpha_{2}<\ldots<\alpha_{r-1}<a_{r} .
$$

Corollary. If $f_{1}(x)=g(x), f_{i}(x)=x^{r-i}$ for $i=2, \ldots, r-1$, then

$$
\frac{E\left(a_{1}, \ldots, a_{r}\right)}{V\left(a_{1}, \ldots, a_{r}\right)}=\frac{1}{(r-1) !} g^{(r-1)}(\xi)
$$

for some $\xi$ in $a_{1}<\xi<a_{r}$.

Pr o o f. Consider $a_{2}, \ldots, a_{r}$ as fixed, $a_{1}$ as variable. Since

$$
E\left(a_{2}, a_{2}, \ldots, a_{r}\right)=V\left(a_{2}, a_{2}, \ldots, a_{r}\right)=0,
$$

Cauchy's mean value theorem gives

$$
\frac{E\left(a_{1}, \ldots, a_{r}\right)}{V\left(a_{1}, \ldots, a_{r}\right)}=\left.\frac{\partial / \partial a_{1} E\left(a_{1}, \ldots, a_{r}\right)}{\partial / \partial a_{1} V\left(a_{1}, \ldots, a_{r}\right)}\right|_{a_{1}=\alpha_{1}} .
$$

We repeat the process with $\alpha_{1}, a_{3}, \ldots, a_{r}$ fixed and $a_{2}$ varying to get

$$
\frac{E\left(a_{1}, \ldots, a_{r}\right)}{V\left(a_{1}, \ldots, a_{r}\right)}=\left.\frac{\partial^{2} / \partial a_{1} \partial a_{2} E\left(a_{1}, \ldots, a_{r}\right)}{\partial^{2} / \partial a_{1} \partial a_{2} V\left(a_{1}, \ldots, a_{r}\right)}\right|_{a_{1}=\alpha_{1}, a_{2}=\alpha_{2}} .
$$

After $r-3$ further steps of this type, we have replaced $E\left(a_{1}, \ldots, a_{r}\right)$ and $V\left(a_{1}, \ldots, a_{r}\right)$ by determinants with all entries zero in the last column except for the bottom entry, which is one. These determinants are equal to the minor determinants of their first $r-1$ rows and columns. In the denominator we have $V\left(\alpha_{1}, \ldots, \alpha_{r-1}\right)$ with the $i$ th column multiplied by $r-i$ for each $i$. This proves the lemma.

For the Corollary we have

$$
E^{\prime}\left(\alpha_{1}, \ldots, \alpha_{r-1}\right)=\left|\begin{array}{ccccc}
g_{1}^{\prime}\left(\alpha_{1}\right) & \alpha_{1}^{r-2} & \ldots & \alpha_{1} & 1 \\
\ldots \ldots & \ldots & \ldots & \ldots & \ldots \\
g_{1}^{\prime}\left(\alpha_{r-1}\right) & \alpha_{r-1}^{r-2} & \ldots & \alpha_{r-1} & 1
\end{array}\right|,
$$

and we obtain the Corollary by iteration. 
In our second lemma we estimate a particular determinant of this type, which is proportional to the difference of the cross-ratios $(a, b ; c, d)$ and $(f(a), f(b) ; f(c), f(d))$.

LEMMA 3.2 (cross-ratio and Schwarzian derivative). Suppose that $f(x)$ is a real function three times continuously differentiable on an interval $I$, and that $f^{\prime \prime}(x)$ is non-zero on $I$, with

$$
\max \left|f^{\prime \prime}(x)\right| \leq B \min \left|f^{\prime \prime}(x)\right| .
$$

For $a<b<c<d$ in I, let

$$
E(a, b, c, d)=\left|\begin{array}{llll}
a f(a) & f(a) & a & 1 \\
b f(b) & f(b) & b & 1 \\
c f(c) & f(c) & c & 1 \\
d f(d) & f(d) & d & 1
\end{array}\right|
$$

Then

$$
\frac{E(a, b, c, d)}{V(a, b, c, d)}=\frac{C}{12}\left(3 f^{\prime \prime}(\xi)^{2}-2 f^{\prime}(\xi) f^{(3)}(\xi)\right)
$$

for some $\xi$ in $a<\xi<d$ and some $C$ in $1 / B^{2} \leq C \leq B^{2}$.

Pr o of. We use Lemma 3.1 twice, with

$$
\begin{aligned}
E^{\prime}(\alpha, \beta, \gamma) & =\left|\begin{array}{lll}
f(\alpha)+\alpha f^{\prime}(\alpha) & \alpha & 1 \\
f(\beta)+\beta f^{\prime}(\beta) & \beta & 1 \\
f(\gamma)+\gamma f^{\prime}(\gamma) & \gamma & 1
\end{array}\right|, \\
E^{\prime \prime}(u, v) & =\left|\begin{array}{ll}
2 f^{\prime}(u)+u f^{\prime \prime}(u) & f^{\prime \prime}(u) \\
2 f^{\prime}(v)+v f^{\prime \prime}(v) & f^{\prime \prime}(v)
\end{array}\right| \\
& =f^{\prime \prime}(u) f^{\prime \prime}(v)\left(\frac{2 f^{\prime}(u)}{f^{\prime \prime}(u)}+u-\frac{2 f^{\prime}(v)}{f^{\prime \prime}(v)}-v\right),
\end{aligned}
$$

whilst $V(u, v)=u-v$.

Again by Cauchy's mean value theorem

$$
\frac{1}{u-v}\left(\frac{2 f^{\prime}(u)}{f^{\prime \prime}(u)}+u-\frac{2 f^{\prime}(v)}{f^{\prime \prime}(v)}-v\right)=3-\frac{2 f^{\prime}(\xi) f^{(3)}(\xi)}{f^{\prime \prime}(\xi)^{2}}
$$

for some $\xi$ in $u<\xi<v$. Thus

$$
\frac{E(a, b, c, d)}{V(a, b, c, d)}=\frac{1}{6 \cdot 2} \cdot \frac{f^{\prime \prime}(u) f^{\prime \prime}(v)}{f^{\prime \prime}(\xi)^{2}}\left(3 f^{\prime \prime}(\xi)^{2}-2 f^{\prime}(\xi) f^{(3)}(\xi)\right),
$$

which gives the result of the lemma.

We note that the condition $f^{\prime \prime}(x) \neq 0$ in Lemma 3.2 is necessary, because for $f(x)=\sin x$, the determinant $E(a, b, c, d)$ can be zero by periodicity, but

$$
3 f^{\prime \prime}(\xi)^{2}-2 f^{\prime}(\xi) f^{(3)}(\xi)=3 \sin ^{2} \xi+2 \cos ^{2} \xi \geq 2 .
$$


On the other hand, Lemma 3.2 holds with $C=1$ for $f(x)=1, x, x^{2}, x^{3}$, $1 / x, 1 / x^{2}$; this can be checked directly.

LEMma 3.3 (interval of approximation). Let $f(x)$ be a real function three times continuously differentiable on an interval $J$ of length $K$, with

$$
\left|f^{(i)}(x)\right| \leq C^{i+1}
$$

for $i=1,2,3$, and

$$
\begin{gathered}
\left|f^{\prime \prime}(x)\right| \geq 1 / C^{2}, \\
\left|3 f^{\prime \prime}(\xi)^{2}-2 f^{\prime}(\xi) f^{(3)}(\xi)\right| \geq 1 / C^{6} .
\end{gathered}
$$

Suppose that $f(x)$ has a Padé approximation

$$
g(x)=\frac{\alpha x+\beta}{\gamma x+\delta}
$$

with

$$
|f(x)-g(x)| \leq \Delta \leq 1 /\left(27 C^{3}\right)
$$

on $I$. Then

$$
K \leq 18 C^{20 / 3} \Delta^{1 / 3} .
$$

Proof. In Lemma 3.2 we take $a$ and $d$ to be the endpoints of $J$, and $d-c=c-b=b-a=K / 3$. Then

$$
|E(a, b, c, d)| \geq \frac{K^{6}}{729 C^{18}} .
$$

We put $f(a)=g(a)+\varepsilon, f(b)=g(b)+\zeta, f(c)=g(c)+\eta, f(d)=g(d)+\theta$, and we expand the determinant. The terms which do not involve $\varepsilon, \zeta, \eta$, or $\theta$ give the corresponding determinant with $f(x)$ replaced by $g(x)$, which is easily seen to be zero by direct calculation.

There are four determinants of the type

$$
\begin{aligned}
\left|\begin{array}{cccc}
a \varepsilon & \varepsilon & 0 & 0 \\
b f(b) & f(b) & b & 1 \\
c f(c) & f(c) & c & 1 \\
d f(d) & f(d) & d & 1
\end{array}\right| & =-\varepsilon\left|\begin{array}{ccc}
(b-a) f(b) & b & 1 \\
(c-a) f(c) & c & 1 \\
(d-a) f(d) & d & 1
\end{array}\right| \\
& =\left.\varepsilon(c-b)(d-b)(d-c) \frac{1}{2} \frac{d^{2}}{d x^{2}}(x-a) f(x)\right|_{x=\xi} \\
& =\varepsilon(c-b)(d-b)(d-c)\left(f^{\prime}(\xi)+\frac{1}{2}(\xi-a) f^{\prime \prime}(\xi)\right)
\end{aligned}
$$

for some $\xi$ in $b<\xi<d$, by the Corollary to Lemma 3.1. Each determinant has absolute value at most

$$
\frac{2 \Delta K^{3}}{9}\left(C^{2}+\frac{C^{3} K}{2}\right) .
$$


There are six determinants of the type

$$
\left|\begin{array}{cccc}
a \varepsilon & \varepsilon & 0 & 0 \\
b \zeta & \zeta & 0 & 0 \\
c f(c) & f(c) & c & 1 \\
d f(d) & f(d) & d & 1
\end{array}\right|=\varepsilon \zeta(b-a)(d-c)
$$

Each such determinant has absolute value at most $\Delta^{2} K^{2} / 9$. We deduce that

$$
\frac{K^{6}}{729 C^{18}} \leq \frac{8 C^{2} \Delta K^{3}}{9}+\frac{4 C^{3} \Delta K^{4}}{9}+\frac{2 \Delta^{2} K^{2}}{3} .
$$

If the first term on the right of (3.8) dominates, then

$$
K \leq 18 C^{20 / 3} \Delta^{1 / 3} .
$$

If the second term on the right of (3.8) dominates, then

$$
K \leq 18 \sqrt{3} C^{21 / 2} \sqrt{\Delta} \text {. }
$$

If the third term on the right of (3.8) dominates, then

$$
K \leq 9 \sqrt{6} C^{9} \sqrt{\Delta} .
$$

By the upper bound for $\Delta$ in (3.6), we see that (3.9) is the strongest of the three conditions.

LEMMA 3.4 (intersection number). Let $f(x)$ be a real function three times continuously differentiable that satisfies the conditions (3.2), (3.3) and (3.4) of Lemma 3.3. Suppose that $f(x)$ has a Padé approximation $g(x)$ of the form (3.5). Then there are at most four disjoint subintervals on which the inequality (3.6) holds.

Proof. If $f(x)=g(x)+e$ for four distinct values $x=a, b, c, d$, then the determinant $E(a, b, c, d)$ of Lemma 3.2 is zero, and (3.1) of Lemma 3.2 contradicts the assumption (3.4). Hence $f(x)-g(x)$ takes each value at most three times. The endpoints of intervals on which (3.6) holds are either $\pm \infty$ or finite values of $x$ at which $f(x)-g(x)= \pm \Delta$. There are at most eight endpoints, so at most four intervals.

LEMMA 3.5 (growth of approximation error). Under the hypotheses of Lemma 3.3, let e be a point outside $J$, on the opposite side of $J$ from the pole of $g(x)$ at $x=-\delta / \gamma$, with

$$
|f(e)-g(e)|=\Delta^{\prime} \geq 2^{6} 3^{6} \Delta .
$$

Let $K^{\prime}$ be the distance of e from the furthest point of the interval $J$. Then

$$
K^{\prime} \geq\left(\frac{\Delta^{\prime}}{\Delta}\right)^{1 / 3} \frac{K}{42 C^{8}} .
$$

Proof. We write $h(x)=f(x)-g(x)$. The denominator $\gamma x+\delta$ has constant sign on $J$; by changing the signs of $\alpha, \beta, \gamma$, and $\delta$, and the sign 
of $x$, we can suppose that $\gamma>0$ and $\gamma x+\delta>0$ on $J$. Let $J$ be the interval $[c, b]$, and let

$$
a=\max \left(c, \frac{1}{2}\left(b-\frac{\delta}{\gamma}\right)\right) .
$$

Let $J^{\prime}$ be the interval $[a, b]$, a subinterval of $J$ with length at least $K / 2$.

We consider two divided differences. Let $d=(2 a+b) / 3, d^{\prime}=(a+2 b) / 3$. We have for some $\xi$ in $a<\xi<b$

$$
\begin{aligned}
\frac{1}{6} h^{(3)}(\xi)= & h\left[a, d, d^{\prime}, b\right] \\
= & \frac{h(a)}{(a-d)\left(a-d^{\prime}\right)(a-b)}+\frac{h(d)}{(d-a)\left(d-d^{\prime}\right)(d-b)} \\
& +\frac{h\left(d^{\prime}\right)}{\left(d^{\prime}-a\right)\left(d^{\prime}-d\right)\left(d^{\prime}-b\right)}+\frac{h(b)}{(b-a)(b-d)\left(b-d^{\prime}\right)} .
\end{aligned}
$$

Thus

$$
\left|h^{(3)}(\xi)\right| \leq 6 \Delta\left(2 \cdot \frac{6}{K} \cdot \frac{3}{K} \cdot \frac{2}{K}+2 \cdot \frac{6}{K} \cdot \frac{6}{K} \cdot \frac{3}{K}\right)=\frac{1728 \Delta}{K^{3}} .
$$

We have

$$
h^{(3)}(\xi)=f^{(3)}(\xi)-\frac{6(\alpha \delta-\beta \gamma) \gamma^{2}}{(\gamma \xi+\delta)^{4}}
$$

SO

$$
\begin{aligned}
\left|\frac{6(\alpha \delta-\beta \gamma) \gamma^{2}}{(\gamma a+\delta)^{4}}\right| & \leq 16\left|\frac{6(\alpha \delta-\beta \gamma) \gamma^{2}}{(\gamma \xi+\delta)^{4}}\right| \leq 16\left|f^{(3)}(\xi)\right|+16\left|h^{(3)}(\xi)\right| \\
& \leq 16 C^{4}+\frac{16 \cdot 1728 \Delta}{K^{3}} \leq \frac{2^{9} 3^{4} 5 C^{24} \Delta}{K^{3}}
\end{aligned}
$$

where we have used the bound (3.7) for $K$.

Secondly, for some $\eta$,

$$
\begin{aligned}
\frac{1}{6} h^{(3)}(\eta)= & h\left[a, d, d^{\prime}, e\right] \\
= & \frac{h(a)}{(a-d)\left(a-d^{\prime}\right)(a-e)}+\frac{h(d)}{(d-a)\left(d-d^{\prime}\right)(d-e)} \\
& +\frac{h\left(d^{\prime}\right)}{\left(d^{\prime}-a\right)\left(d^{\prime}-d\right)\left(d^{\prime}-e\right)}+\frac{h(e)}{(e-a)(b-e)\left(e-d^{\prime}\right)} .
\end{aligned}
$$

Now

$$
e-d^{\prime} \geq K^{\prime}-2 K / 3 \geq K^{\prime} / 3, \quad e-d \geq K^{\prime}-K / 3 \geq 2 K^{\prime} / 3 .
$$


Since $|h(e)|=\Delta^{\prime}$, we have

$$
\begin{aligned}
\left|\frac{h^{(3)}(\eta)}{6}\right| & \geq \frac{\Delta^{\prime}}{K^{\prime 3}}-\Delta\left(\frac{6}{K} \cdot \frac{3}{K} \cdot \frac{1}{K^{\prime}}+\frac{6}{K} \cdot \frac{6}{K} \cdot \frac{3}{2 K^{\prime}}+\frac{3}{K} \cdot \frac{6}{K} \cdot \frac{3}{K^{\prime}}\right) \\
& =\frac{\Delta^{\prime}}{K^{\prime 3}}-\frac{126 \Delta}{K^{2} K^{\prime}} .
\end{aligned}
$$

Again

$$
h^{(3)}(\eta)=f^{(3)}(\eta)-\frac{6(\alpha \delta-\beta \gamma) \gamma^{2}}{(\gamma \eta+\delta)^{4}}
$$

SO

$$
\begin{aligned}
\left|\frac{6(\alpha \delta-\beta \gamma) \gamma^{2}}{(\gamma a+\delta)^{4}}\right| & \geq\left|\frac{6(\alpha \delta-\beta \gamma) \gamma^{2}}{(\gamma \eta+\delta)^{4}}\right| \geq\left|h^{(3)}(\eta)\right|-\left|f^{(3)}(\eta)\right| \\
& \geq \frac{6 \Delta^{\prime}}{K^{\prime 3}}-\frac{756 \Delta}{K^{2} K^{\prime}}-C^{4} \\
& \geq \frac{6 \Delta^{\prime}}{K^{\prime 3}}-\frac{2^{2} 3^{3} 7 \Delta}{K^{2} K^{\prime}}-\frac{2^{3} 3^{6} C^{24} \Delta}{K^{2} K^{\prime}}
\end{aligned}
$$

by (3.7). Comparing (3.12) and (3.13), we see that

$$
\frac{2^{4} 3^{5} 5 \cdot 11 C^{24} \Delta}{K^{3}} \geq \frac{6 \Delta^{\prime}}{K^{\prime 3}}-\frac{2^{2} 3^{3} 7 \Delta}{K^{2} K^{\prime}} .
$$

Hence either

$$
\frac{K^{\prime}}{K} \geq \frac{1}{42 C^{8}}\left(\frac{\Delta^{\prime}}{\Delta}\right)^{1 / 3},
$$

or

$$
\frac{K^{\prime}}{K} \geq \frac{1}{216}\left(\frac{\Delta^{\prime}}{\Delta}\right)^{1 / 2} \geq \frac{1}{36}\left(\frac{\Delta^{\prime}}{\Delta}\right)^{1 / 3},
$$

and (3.14) is the weaker conclusion.

4. Major arcs. A major arc is an interval $J$ on which there are at least four points of $S$, and all points of $S$ on $J$ have $r / q=G(m / n)$ for some linear fractional function $G(x)$.

LEMMA 4.1 (divisibility). The equation of a non-constant major arc $J$ can be written as

$$
G(x)=\frac{a x+b}{c x+d},
$$

where $a, b, c, d$ are integers with highest common factor $(a, b, c, d)=1$. If $\left(m_{i} / n_{i}, r_{i} / q_{i}\right)$ is a point of $S$ on $J$, then the highest common factor

$$
e_{i}=\left(a m_{i}+b n_{i}, c m_{i}+d n_{i}\right)
$$


is a factor of $|a d-b c|$, and if two points of $S$ on $J$ have $e_{i}=e_{j}=e$, then $e \mid\left(m_{i} n_{j}-m_{j} n_{i}\right)$, so

$$
\left|\frac{m_{i}}{n_{i}}-\frac{m_{j}}{n_{j}}\right| \geq \frac{e}{M^{2}} .
$$

Proof. If $\left(m_{i} / n_{i}, r_{i} / q_{i}\right), i=1, \ldots, 4$, are four distinct points of $S$ on $J$, then

$$
\left|\begin{array}{llll}
m_{1} q_{1} & m_{1} r_{1} & n_{1} q_{1} & n_{1} r_{1} \\
m_{2} q_{2} & m_{2} r_{2} & n_{2} q_{2} & n_{2} r_{2} \\
m_{3} q_{3} & m_{3} r_{3} & n_{3} q_{3} & n_{4} r_{4} \\
m_{4} q_{4} & m_{4} r_{4} & n_{4} q_{4} & n_{4} r_{4}
\end{array}\right|=0 .
$$

Let $A,-B, C,-D$ be the cofactors of the first row. Then $A, B, C, D$ are integers with

$$
A m_{i} q_{i}-B m_{i} r_{i}+C n_{i} q_{i}-D n_{i} r_{i}=0 .
$$

If $A, B, C, D$ are all zero, then we consider cofactors of the first row in the determinant

$$
B=\left|\begin{array}{lll}
m_{2} q_{2} & n_{2} q_{2} & n_{2} r_{2} \\
m_{3} q_{3} & n_{3} q_{3} & n_{3} r_{3} \\
m_{4} q_{4} & n_{4} q_{4} & n_{4} r_{4}
\end{array}\right| .
$$

Since $m_{3} / n_{3} \neq m_{4} / n_{4}$, the cofactor of $n_{2} r_{2}$ is non-zero. There is a relation of the form (4.3), but with $B=0, D \neq 0$. Having found a relation with $A$, $B, C, D$ not all zero, we obtain the integers $a, b, c, d$ by dividing $A, B, C$, $D$ by the highest common factor $(A, B, C, D)$.

For $(m / n, r / q)$ on the minor arc $J$, we define

$$
e=(a m+b n, c m+d n),
$$

so that

$$
\begin{array}{cl}
a m+b n=e r, & c m+d n=e q, \\
e(a q-c r)=(a d-b c) n, \quad & e(d r-b q)=(a d-b c) m .
\end{array}
$$

Since $(m, n)=1$, we have $e \mid(a d-b c)$. If $m_{i} / n_{i}, m_{j} / n_{j}$ correspond to the same $e$ in (4.4), then

$$
e \mid a\left(m_{i} n_{j}-m_{j} n_{i}\right),
$$

and similarly with $a$ replaced by $b, c$, or by $d$. Since the highest common factor $(a, b, c, d)$ is unity, we deduce that $e \mid\left(m_{i} n_{j}-m_{j} n_{i}\right)$. Hence

$$
\left|\frac{m_{i}}{n_{i}}-\frac{m_{j}}{n_{j}}\right| \geq \frac{e}{n_{i} n_{j}} \geq \frac{e}{M^{2}},
$$

which completes the proof of the lemma. 
LEMMA 4.2 (orders of magnitude). Under the hypotheses of Lemma 1.1, and in the notation (4.1), on a major arc,

$$
|a d-b c| \leq T=18\left(2 C^{2} \lambda+1\right)^{3} M^{6} Q^{6},
$$

where $C$ is the constant in Theorem 1 . If $a d-b c=0$, then $e=1$. If $a d-b c \neq 0$, then there are at most $\tau$ values for $e$, where

$$
\tau=\max _{t \leq T} d(t)=O\left(\left(C \lambda M Q^{2}\right)^{\varepsilon}\right)
$$

for any $\varepsilon>0$, with implied constant depending on $\varepsilon$.

Proof. As in Lemma 1.1, the rational numbers $r / q$ lie in some interval $k \leq r / q \leq k+K$, where $k$ and $K$ are integers with

$$
K \leq 2 C^{2} \lambda+1 .
$$

If we replace $y$ by $y-k$ in (4.1), then $a$ and $b$ change to integer values $a^{\prime}$, $b^{\prime}$ with $a^{\prime} d-b^{\prime} c=a d-b c$. Since $a^{\prime}, b^{\prime}, c, d$ are factors of cofactors in the determinant (4.2), we have

$$
\left|a^{\prime}\right|,|c| \leq 3 K^{2} M^{3} Q^{3}, \quad\left|b^{\prime}\right|,|d| \leq 3 K M^{3} Q^{3},
$$

which gives (4.5). In the constant case $e=(q, r)=1$. In the non-constant case $e$ is a factor of a positive integer $|a d-b c| \leq T$, and we use the standard estimate for the divisor function.

In order to use Lemma 3.5, we define a proper major arc to be an interval $J$ on the $x$-line on which

$$
|F(x)-G(x)| \leq \delta / Q^{2},
$$

where $G(x)$ has the linear fractional form (4.1), and all points of $S$ on $J$ have $r / q=G(m / n)$. By Lemma 3.4, a major arc decomposes into at most four proper major arcs.

Lemma 4.3 (spacing of major arcs). Suppose that all points of $S$ have

$$
M / 2 \leq m \leq M .
$$

Let $J$ be a proper major arc, of length $K$, containing $R(J)$ points of $S$. Suppose that $f(x)=F(x) / \lambda$ satisfies the conditions of Lemma 3.3, with

$$
\Delta=\frac{\delta}{\lambda Q^{2}} \leq \frac{1}{27 C^{3}} .
$$

Then either

$$
R(J) \leq \tau,
$$

or there is an interval $J^{\prime}$ of length $K^{\prime}$ containing $J$ (the interval $J^{\prime}$ may extend outside $I$ ) such that all points of $S$ in $J^{\prime}$ lie in $J$, and

$$
R(J)=O\left(C^{6} \delta^{1 / 4} K^{\prime} M^{2} \tau\right) .
$$


Proof. There are at most $\tau$ different values of the common factor $e$. If some value of $e$ occurs twice, let $A(e)$ be the set of points of $S$ on $J$ with this value of $e$, let $J(e)$, of length $K(e)$, be the shortest subinterval of $J$ that contains $A(e)$. Let $m / n$ be the endpoint of $J(e)$ furthest from the pole $x=-d / c$. Then

$$
\left|\frac{m}{n}+\frac{d}{c}\right| \geq K(e)
$$

and

$$
a m+b n=e r, \quad c m+d n=e q .
$$

Now let $\left(m^{\prime} / n^{\prime}, r^{\prime} / q^{\prime}\right)$ be a point of $S$ with $r^{\prime} / q^{\prime} \neq G\left(m^{\prime} / n^{\prime}\right)$, and with $m^{\prime} / n^{\prime}$ on the opposite side of the minor arc $J$ to the pole at $x=-d / c$. Let

$$
K^{\prime}=\max _{x \in J}\left|\frac{m^{\prime}}{n^{\prime}}-x\right| \text {. }
$$

Then by (4.7),

$$
\frac{c m^{\prime}+d n^{\prime}}{c m+d n}=\frac{n^{\prime}\left(m^{\prime} / n^{\prime}+d / c\right)}{n(m / n+d / c)} \leq \frac{2 K^{\prime}}{K(e)},
$$

so

$$
\begin{gathered}
c m^{\prime}+d n^{\prime} \leq \frac{2 e K^{\prime} Q}{K(e)}, \\
\left|\frac{r^{\prime}}{q^{\prime}}-\frac{a m^{\prime}+b n^{\prime}}{c m^{\prime}+d n^{\prime}}\right| \geq \frac{1}{\left(c m^{\prime}+d n^{\prime}\right) q^{\prime}} \geq \frac{K(e)}{2 e K^{\prime} Q^{2}} .
\end{gathered}
$$

We deduce that if

$$
K^{\prime} \leq \frac{K(e)}{4 \delta e}
$$

then

$$
\left|F\left(\frac{m^{\prime}}{n^{\prime}}\right)-\frac{a m^{\prime}+b n^{\prime}}{c m^{\prime}+d n^{\prime}}\right| \geq \frac{K(e)}{2 e K^{\prime} Q^{2}}-\frac{\delta}{Q^{2}} \geq \frac{K(e)}{4 e K^{\prime} Q^{2}} .
$$

We apply Lemma 3.5 with

$$
\Delta^{\prime} \geq \frac{K(e)}{4 e K^{\prime} \lambda Q^{2}} .
$$

If

$$
\Delta^{\prime}<2^{6} 3^{6} \Delta
$$

then

$$
\frac{K^{\prime}}{K(e)} \geq \frac{1}{2^{8} 3^{6} \delta e} .
$$

We note that (4.13) also holds when (4.11) is false. 
If (4.12) is false, then Lemma 3.5 is valid, and

$$
\frac{K^{\prime}}{K(e)} \geq \frac{1}{42 C^{8}}\left(\frac{\Delta^{\prime}}{\Delta}\right)^{1 / 3}=\frac{1}{42 C^{8}}\left(\frac{1}{4 \delta e} \cdot \frac{K(e)}{K^{\prime}}\right)^{1 / 3},
$$

so

$$
\frac{K^{\prime}}{K(e)} \geq \frac{1}{\left(2^{5} 3^{7} 7^{3} C^{24} \delta e\right)^{1 / 4}} .
$$

The number of points in $A(e)$ is

$$
\begin{aligned}
& \leq \frac{K(e) M^{2}}{e}+1 \leq \frac{2 K(e) M^{2}}{e} \\
& \leq 2 M^{2} K^{\prime} \max \left(2^{8} 3^{6} \delta,\left(2^{9} 3^{3} 7^{3} C^{24} \delta e\right)^{1 / 4}\right) .
\end{aligned}
$$

Hence $R(J)$, the number of points of $S$ in $J$, is bounded by the sum over $e$ of the maximum of one and the expression in (4.14). There are at most $\tau$ values for $e$ by Lemma 4.2, so one of the estimates (4.9) or (4.10) holds.

Proof of Theorem 1. We can suppose that $\delta \leq 1 / C^{24}$, or the result is trivial. By (1.6), the hypothesis (4.8) of Lemma 4.3 holds. We make the temporary assumption that all points $(\mathrm{m} / \mathrm{n}, \mathrm{r} / \mathrm{q})$ of $S$ satisfy (4.7) of Lemma 4.3. By Lemma 2.4, the points of $S$ lie in $O\left(L / K_{0}\right)$ intervals, each containing either an isolated point or a major arc. Hence minor arcs and proper major arcs with at most $\tau$ points of $S$ contribute

$$
O\left(\frac{L T}{K_{0}}\right)=O\left(\frac{1}{K_{0}}\left(C \lambda M Q^{2}\right)^{\varepsilon}\right)
$$

points to $R$. This is the second term in (1.7).

Other minor arcs can be embedded in intervals in which the density of points of $S$ is $O\left(C^{6} \delta^{1 / 4} \tau\right)$ by the case (4.10) of Lemma 4.3. At most two such intervals overlap at each point (eight intervals if we count proper major arcs separately), and at most two such intervals extend outside $I$. By Lemma 3.3, the intervals that extend outside $I$ correspond to at most eight proper major arcs of length

$$
O\left(\left(\frac{C^{20} \delta}{\lambda Q^{2}}\right)^{1 / 3}\right)
$$

which contribute

$$
O\left(\left(\frac{C^{20} \delta}{\lambda Q^{2}}\right)^{1 / 3} M^{2}\right)
$$

points to $S$. The total length of all the other intervals is $O(1)$, and they contribute

$$
O\left(C^{6} \delta^{1 / 4} M^{2} \tau\right)
$$


points to $S$. Since $\delta \leq 1 / C^{24}$, the term (4.16) absorbs (4.15); it is the first term in (1.7).

We complete the proof of Theorem 1 by summing ranges $M_{i} \leq m \leq$ $M_{i-1}$, where $M_{i-1} \leq 2 M_{i}$, and $M_{0}=M$. The largest range $i=1$ dominates, and the sum over $i$ affects only the constant in (1.7).

\section{References}

[1] M. Filaseta and O. Trifonov, The distribution of fractional parts with applications to gap results in number theory, Proc. London Math. Soc. (3) 73 (1996), 241-278.

[2] H. Halberstam and K. F. Roth, On the gaps between consecutive $k$-free numbers, J. London Math. Soc. 26 (1951), 268-273.

[3] M. N. Huxley, The integer points close to a curve, Mathematika 36 (1989), 198-215.

[4] -, The rational points close to a curve, Ann. Scuola Norm. Sup. Pisa Cl. Sci. Fis. Mat. (4) 21 (1994), 357-375.

[5] -, Moments of differences between square-free numbers, in: Sieve Methods, Exponential Sums and their Applications in Number Theory, G. R. H. Greaves, G. Harman and M. N. Huxley (eds.), Cambridge Univ. Press, 1996, 187-204.

[6] M. N. Huxley et P. Sargos, Points entiers au voisinage d'une courbe plane de classe $C^{n}$, Acta Arith. 69 (1995), 359-366.

[7] H. P. F. Swinnerton-Dyer, The number of lattice points on a convex curve, J. Number Theory 6 (1974), 128-135.

School of Mathematics

University of Cardiff

23 Senghenydd Road

Cardiff CF24 4YH

Wales, Great Britain

E-mail: huxley@cardiff.ac.uk 